\title{
Geographic Genetic Contour of a Ground Beetle, Scarites aterrimus (Coleoptera: Carabidae) on the Basis of Mitochondrial DNA Sequence
}

\author{
Ah Rha Wang', Min Jee Kim ${ }^{1}$, Young Bok $\mathrm{Cho}^{2}$, Xinlong Wan, and Iksoo Kim ${ }^{1 \text {,* }}$ \\ ${ }^{1}$ College of Agriculture \& Life Sciences, Chonnam National University, Gwangju 500-757, Korea \\ ${ }^{2}$ Natural History Museum, Han Nam University, Daejeon 306-791, Korea \\ (Received 28 March 2011; Accepted 21 April 2011)
}

The Scarites aterrimus (Coleoptera: Carabidae), is one of the carabid beetles dwelling exclusively on coastal sandy dunes. Habitat deterioration and equivalent activity have greatly concerned population declines in several species dwelling on the coastal sandy dunes. As a first step to establish long-term conservation strategy, we investigated the nation-wide magnitude and nature of genetic diversity of the species. As a first step, we sequenced a portion of mitochondrial COI gene, corresponding to "DNA Barcode" region (658 bp) from a total of $24 \mathrm{~S}$. aterrimus individuals collected over nine sandy dunes belonging to four Korean provinces. The sequence analysis evidenced moderate to low magnitude of sequence diversity compared with other insect species distributed in Korean peninsula $(0.152 \%$ to $0.912 \%)$. The presence of closely related haplotypes and relatively high gene flow estimate collectively suggest that there had been no historical barriers that bolster genetic subdivision. Population decline was postulated on the basis of several missing haplotypes that are well found in the species with a large population size. This interpretation is consistent with field observation of small population size in the coastal sandy dune habitats. The highest genetic diversity estimates were found in the coastal sand dune population of Seogwipo, Jeju Island, justifying a prior attention to the population, in order to sustain overall genetic diversity of the species. Further scrutinized study might be required for further robust conclusion.

\footnotetext{
*To whom the correspondence addressed

College of Agriculture and Life Sciences, Chonnam National University, Gwangju 500-757, Korea.

Tel: +82-62-530-2073; Fax: +82-62-530-2079;

E-mail: ikkim81@chonnam.ac.kr
}

Key words: Mitochondrial DNA, COI gene, Scarites aterrimus, Ground beetle, Carabid Population genetic structure, Conservation genetics

\section{Introduction}

As has been known, world biodiversity in fact has greatly been diminished in several levels, such as community, species, geographic populations, and even in the genetic diversity (Soulé, 1986; Wilson, 1992). The main cause for such diminishment includes a massive destruction of habitat, urbanization, contamination and so on. In this regard, biodiversity in Korea also has greatly been exterminated for over the last 50 years and now more than 200 species (including 20 insect species) are endangered.

The coastal dunes also have long been subjected to construction, deterioration, and fragmentation, although these are important habitat for many wildlife including insects such as carabid beetles (Kim, 1980, 2003). In fact, several species of carabid beetles dwelling on coastal sandy beaches are reported to rarely be found and some of them are under the pressure of extinction. Thus, these species are considered to be the potential endangered species in Korea. In this circumstance, it is necessary to take some steps to rescue the carabid beetles for long-term conservation.

The ground beetle, Scarites aterrimus (Carabidae: Coleoptera), is one of the carabids well adapted to shore environment in littoral (Kawakami et al., 2004). The medium-sized (e.g., $20 \mathrm{~mm}$ in body length) S. aterrimus that occurs from May to September is distributed in sandy beaches in Korea, Japan, and China (Park and Paik, 2001). In Korea and Japan, the great population diminishment has been reported (personal communication, Cho; Kawakami and Sugiura, 2006).

In this study, we, thus, investigated genetic diversity, 
Table 1. A list of trapping localities, animal numbers, mitochondrial COI haplotypes of Scarites aterrimus

\begin{tabular}{|c|c|c|c|c|}
\hline $\begin{array}{l}\text { Collecting locality } \\
\text { (no. of individuals) }\end{array}$ & Collection date & Animal number & $\begin{array}{c}\text { COI } \\
\text { haplotype }\end{array}$ & $\begin{array}{c}\text { Genbank accession } \\
\text { number }\end{array}$ \\
\hline \multirow{3}{*}{$\begin{array}{l}\text { 1. Sand dune of Goraebul, Byeonggok-ri } \\
\text { Gyeongsangbuk-do province (3) }\end{array}$} & 2009.07 .22 & SA3570 & BARSA06 & JF713794 \\
\hline & 2009.07 .22 & SA3571 & BARSA03 & JF713795 \\
\hline & 2009.07 .22 & SA3572 & BARSA02 & JF713796 \\
\hline $\begin{array}{l}\text { 2. Sand dune of Dukcheon, Dukcheon-ri } \\
\text { Gyeongsangbuk-do province (1) }\end{array}$ & 2009.05 .24 & SA3573 & BARSA03 & JF713797 \\
\hline $\begin{array}{l}\text { 3. Sand dune of Songji, Songho-ri } \\
\text { Jeollanam-do province (1) }\end{array}$ & 2008.07 .22 & SA3574 & BARSA09 & JF713798 \\
\hline \multirow{2}{*}{$\begin{array}{l}\text { 4. Sand dune of Buldeung, Tongho-ri } \\
\text { Jeollanam-do province (2) }\end{array}$} & 2008.07 .22 & SA3575 & BARSA03 & JF713799 \\
\hline & 2009.07 .23 & SA3576 & BARSA03 & JF713800 \\
\hline \multirow{3}{*}{$\begin{array}{l}\text { 5. Sand dune of Namryul, Namryul-ri } \\
\text { Jeollanam-do province (3) }\end{array}$} & 2009.05 .23 & SA3577 & BARSA03 & JF713801 \\
\hline & 2009.05 .23 & SA3578 & BARSA03 & JF713802 \\
\hline & 2009.05 .23 & SA3579 & BARSA03 & JF713803 \\
\hline \multirow{6}{*}{$\begin{array}{l}\text { 6. Sand dune of Sohwang, Sohwang-ri } \\
\text { Chungcheongnam-do province (6) }\end{array}$} & 2008.07 .15 & SA3580 & BARSA03 & JF713804 \\
\hline & 2008.07 .15 & SA3581 & BARSA08 & JF713805 \\
\hline & 2009.05 .22 & SA3582 & BARSA03 & JF713806 \\
\hline & 2009.05 .22 & SA3583 & BARSA03 & JF713807 \\
\hline & 2009.05 .22 & SA3584 & BARSA03 & JF713808 \\
\hline & 2009.05 .22 & SA3585 & BARSA03 & JF713809 \\
\hline \multirow{4}{*}{$\begin{array}{l}\text { 7. Sand dune of Songseok, Songseok-ri } \\
\text { Jeollanam-do province (4) }\end{array}$} & 2009.05 .22 & SA3587 & BARSA01 & JF713810 \\
\hline & 2009.05 .22 & SA3589 & BARSA03 & JF713811 \\
\hline & 2009.05 .22 & SA3590 & BARSA03 & JF713812 \\
\hline & 2009.05 .22 & SA3591 & BARSA10 & JF713813 \\
\hline $\begin{array}{l}\text { 8. Sand dune of Jangpo, Jangpo-ri } \\
\text { Chungcheongnam-do province (1) }\end{array}$ & 2008.07 .15 & SA3592 & BARSA03 & JF713814 \\
\hline \multirow{3}{*}{$\begin{array}{l}\text { 9. Sand dune of Seogwipo, Seogwipo city } \\
\text { Jeju-do province (3) }\end{array}$} & 2004.05 .18 & SA3593 & BARSA05 & JF7137815 \\
\hline & 2004.05 .18 & SA3594 & BARSA07 & JF7137816 \\
\hline & 2004.05 .18 & SA3595 & BARSA04 & JF7137817 \\
\hline
\end{tabular}

geographic variation, and populations genetic structure of the species collected from several sandy beaches of Korean coasts (East Sea, West Sea, and South Sea) and a remote island Jeju to accumulate fundamental information of the species for conservation purpose. As a first step, we sequenced a partial mitochondrial cytochrome $b$ oxidase subunit I (COI) gene. This molecular maker has been utilized to illustrate several geographic genetic perspectives of insects occurring in Korean peninsula (e.g., Jeong et al., 2009; Kim et al. 2009).

\section{Materials and Methods}

\section{Sampling}

Adult $S$. aterrimus were sampled from nine Korean localities in 2008 and 2009. Sampling locality, number of individuals, date of collection, and GenBank accession numbers for the individuals of each population are provided in Table 1, and the locality map is shown in Fig. 1. The different sampling size may reflect the difference in population size at each locality, considering that similar effort for sample collection was made. The samples were deposited in $70 \%$ alcohol until being used in molecular analysis.

\section{DNA extraction, primer, PCR, and sequencing}

The total DNA was extracted using the Wizard Genomic DNA Purification Kit, in accordance with the manufacturer's instructions (Promega, USA). For the amplification of a portion of mitochondrial COI gene, corresponding to "DNA Barcode" region being utilized for global animal identification (Hebert et al., 2003) a pair of primer designed from Folmer et al. (1994) was applied, but not always successful. Thus, we designed another forward primer, named COLCOIF1, 5'-AAACTAWTARCCTTCAAAG-3' from the alignment of several fulllength mitochondrial genomes of Coleoptera. This primer 


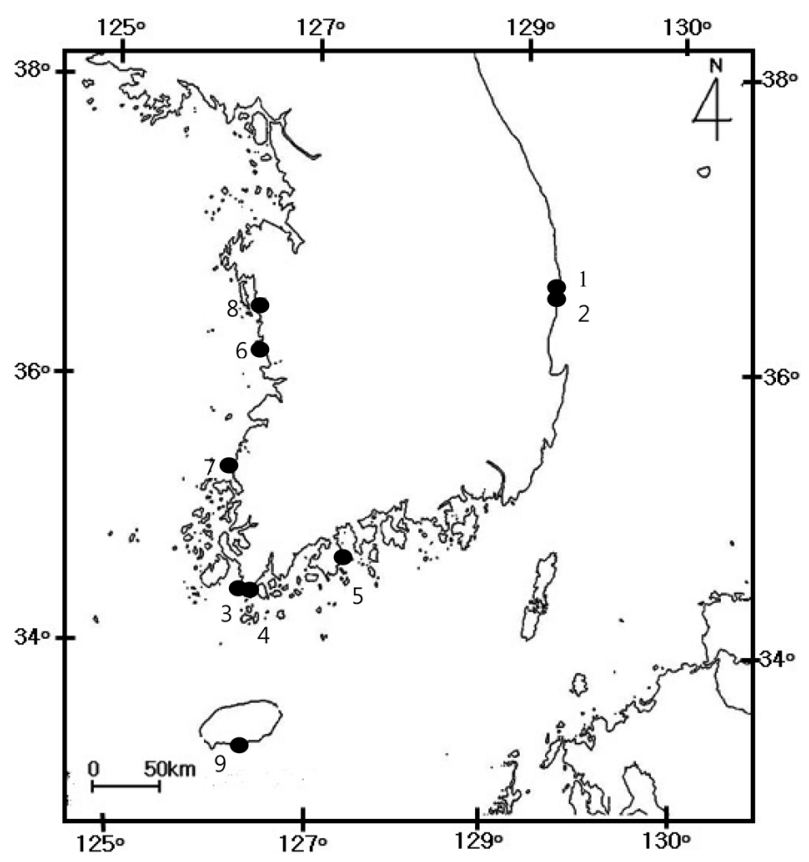

Fig. 1. Sampling location of Scarites aterrimus in Korea. General locality names are as follows: 1, Goraebul, Gyeongsangbukdo; 2, Duckcheon, Gyeongsangbukdo; 3, Songji, Jeollanamdo; 4, Buldeung, Jeollanamdo; 5, Namryul, Jeollanamdo; 6, Sohwang, Cungcheongnamdo; 7, Songseok, Jeollanamdo; 8, Jangpo, Chungcheongnamdo; and 9, Seowipo, Jejudo.

Table 2. List of primers used to amplify and sequence the COI sequences of Scarites aterrimus

\begin{tabular}{ccc}
\hline Fragment & Primer name & Sequence $\left(5^{`}-3^{`}\right)$ \\
\hline \multirow{2}{*}{ F1 } & COLCOIF1 & $\begin{array}{c}\text { AACTAWTARCCT- } \\
\text { TCAAAG }\end{array}$ \\
\cline { 2 - 3 } & ST-INTER-R1 & $\begin{array}{c}\text { GATGATACAC- } \\
\text { CAGCTAAATG }\end{array}$ \\
\hline \multirow{2}{*}{ F2 } & ST-INTER-F1 & $\begin{array}{r}\text { GGGAGCACCAGACAT- } \\
\text { AGC }\end{array}$ \\
\cline { 2 - 3 } & HCO2198 & $\begin{array}{c}\text { TAAACTTCAGGGTGAC- } \\
\text { CAAAAATCA }\end{array}$ \\
\hline
\end{tabular}

amplified the "DNA Barcode" region in combination with the reverse primer, named $\mathrm{HCO} 2198$, 5'-TAAACTTCAGGGTGACCAAAAAATAC-3', which was designed from Folmer et al. (1994). Nevertheless, the efficiency of the amplification was still not perfect enough. Therefore, we additionally designed two overlapping internal primers with different directions after several sequences of COI gene from $S$. aterrimus individuals were obtained. Each of these internal primers was in combination successfully used with each of the universal primers reported in Folmer et al. (1994). The primers information is listed in Table 2. After an initial denaturation step at $94^{\circ} \mathrm{C}$ for $7 \mathrm{~min}$, a 35 - cycle amplification $\left(94^{\circ} \mathrm{C}\right.$ for $1 \mathrm{~min}, 48 \sim 53^{\circ} \mathrm{C}$ for $1 \mathrm{~min}$, and $72^{\circ} \mathrm{C}$ for $1 \mathrm{~min}$ ) was conducted. The final extension step was continued for $7 \mathrm{~min}$ at $72^{\circ} \mathrm{C}$. To confirm the successful DNA amplification, electrophoresis was carried out using $0.5 \times$ TAE buffer on $0.5 \%$ agarose gel. The PCR product was then purified using PCR purification Kit (QIAGEN, Germany). Each fragment of the gene was cloned into a pGEM-T Easy vector (Promega). For the cloning process, XL1-Blue competent cells (Stratagene, USA) were transformed with the ligated DNA, and the resultant plasmid DNA was isolated using a Wizard Plus SV Minipreps DNA Purification System (Promega). DNA sequencing was conducted using the ABI PRISM ${ }^{{ }_{-}}$ BigDye $^{\circledR}$ Terminator ver. 3.1 Cycle Sequencing Kit with an ABI 377 Genetic Analyzer (PE Applied Biosystems, USA). All products were sequenced from both strands.

\section{Sequence and phylogenetic analyses}

Sequence alignment was performed using CLUSTAL X programs (ver. 1.8; Thompson et al., 1997). When homologous sequences from two individuals differed by $\geq$ one nucleotide base, the sequences were considered as different haplotypes. Haplotype designations were applied to new sequences as they were discovered (i.e., BARSA01, BARSA02, BARSA03 and so forth).

Phylogenetic analysis was performed by maximum-parsimony (MP) method (Fitch, 1971) using PAUP* (Phylogenetic Analysis Using Parsimony and Other Method*) ver. 4.0b10 (Swofford, 2002). To root trees, the withinfamilial species of Carabidae, Loricera pilicornis, was utilized as an outgroup (GenBank accession number FN868618). The analysis was performed using an equal weighting of transitions and transversions by heuristic search. The reliability of the trees was tested by 1,000 iterations of bootstrapping (Felsenstein, 1985). With intraspecific mtDNA sequence data it often happens that parsimony analyses provide limited resolution because of polytomies, possibly caused by back mutations and parallel mutations. One solution, which we employed, is to prepare one-step median networks, which provide insight into probable relationships among closely related lineages (Bandelt et al., 1995).

\section{Genetic diversity indices}

For the genetic diversity indices and subsequent population genetic analyses, the populations with more than one haplotype were included in the analyses. Thus, four populations (localities 1, 3, 4, and 6) were selected by this criterion and the remaining populations that were represented either by single individual or single haplotype were excluded from the analyses. Genetic diversity estimates, such as haplotype diversity and nucleotide diver- 
30

BARSA01 BARSA02

BARSA 03

BARSA 04

BARSA 05

BARSA0 6

BARSA0 7

BARSA0 8

BARSA0 9

BARSA 10

BARSA 01

BARSA02

BARSA0 3

BARSA 04

BARSA 05

BARSA0 6

BARSA0 7

BARSA0 8

BARSA09

BARSA10

BARSA01

BARSA02

BARSA0 3

BARSA 04

BARSA 05

BARSA 06

BARSA 07

BARSA0 8

BARSA0 9

BARSA10

BARSA01

BARSA02

BARSA0 3

BARSA 04

BARSA 05

BARSA0 6

BARSA0 7

BARSA0 8

BARSA0 9

BARSA 10

BARSA01

BARSA 02

BARSA0 3

BARSA 04

BARSA 05

BARSA0 6

BARSA 07

BARSA0 8

BARSA0 9

BARSA 10

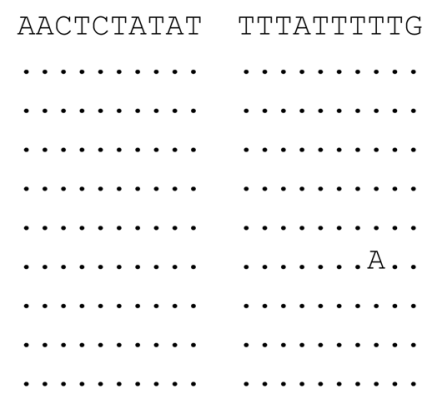

AATTCGAGCA ........

$\ldots \ldots \ldots$

$\ldots \ldots \ldots$

$\ldots \ldots \ldots$

$\ldots \ldots \ldots$

$\ldots \ldots \ldots$

$\ldots \ldots \ldots$

$\ldots \ldots$

$\ldots \ldots \ldots$

TATTGTTACT ........

$\ldots \ldots \ldots$

$\ldots \ldots \ldots$

$\ldots \ldots \ldots$

$\ldots \ldots \ldots$

$\ldots \ldots$

$\ldots \ldots$

........

AGGATTTGGA

..........

$\ldots \ldots \ldots$

$\ldots \ldots \ldots$

$\ldots \ldots$

$\ldots \ldots \ldots$

$\ldots \ldots \ldots$

$\ldots \ldots$

.........

$\ldots \ldots$

$\ldots \ldots$

$\ldots \ldots$.

$\ldots \ldots \ldots$

$\ldots \ldots \ldots$

$\ldots \ldots$

$\ldots \ldots \ldots$

$\ldots \ldots$
........

AATAAATAAT
GAGCATGATC ........

$\ldots \ldots \ldots$

........

$\ldots \ldots \ldots$

$\ldots \ldots \ldots$

$\ldots \ldots \ldots$

$\ldots \ldots \ldots$

$\ldots \ldots \ldots$

$\ldots \ldots \ldots$

90

GAATtAgGAA ATCCTGGATC

$\ldots \ldots$.

$\ldots \ldots \ldots$

$\ldots \ldots \ldots$

$\ldots \ldots \ldots$

$\ldots \ldots \ldots$

$\ldots \ldots \ldots$

$\ldots \ldots \ldots$

$\ldots \ldots \ldots$

GCCCATGCTT

........

$\ldots \ldots \ldots$

$\ldots \ldots \ldots$

$\ldots \ldots \ldots$

$\ldots \ldots \ldots$

$\ldots \ldots \ldots$

$\ldots \ldots \ldots$

$\ldots \ldots \ldots$

$\ldots \ldots \ldots$

AATTGACTAG

.........

$\ldots \ldots \ldots$

$\ldots \ldots . .$.

$\ldots \ldots \ldots$

$\ldots \ldots \ldots$

$\ldots \ldots \ldots$

$\ldots \ldots \ldots$

$\ldots \ldots \ldots$

$\ldots \ldots \ldots$

\section{ATAAGTTTTT}

........

$\ldots \ldots \ldots$

$\ldots \ldots$

$\ldots \ldots \ldots$

$\ldots \ldots$

$\ldots \ldots \ldots$

$\ldots \ldots . .$.

$\ldots \ldots$

$\ldots \ldots$

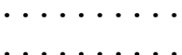

$\ldots \ldots \ldots$

$\ldots \ldots \ldots$

$\ldots \ldots \ldots$

$\ldots \ldots \ldots$

$\ldots \ldots \ldots$

$\ldots \ldots \ldots$

150

TTATTATAAT

\section{........}

$\ldots \ldots$

$\ldots \ldots \ldots$

$\ldots \ldots \ldots$

$\ldots \ldots \ldots$

$\ldots \ldots$

$\ldots \ldots \ldots$

210

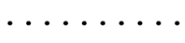

$\ldots \ldots \ldots$

$\ldots \ldots \ldots$

$\ldots \ldots \ldots$

$\ldots \ldots \ldots$

$\ldots \ldots \ldots$

$\ldots \ldots \ldots$

……

GACTTTTACC

$\ldots \ldots \ldots$

$\ldots \ldots \ldots$

...... Т.

$\ldots \ldots \ldots$

$\ldots \ldots \ldots$

$\ldots \ldots \ldots$

$\ldots \ldots \ldots$

$\ldots \ldots \ldots$

$\ldots \ldots$ $\ldots \ldots \ldots$

TTCCTTTGAT

$\ldots \ldots \ldots$ ........
AGGAATAGTA ........

$\ldots \ldots \ldots$

$\ldots \ldots \ldots$

$\ldots G \ldots$.

$\ldots \ldots$

$\ldots \ldots \ldots$

$\ldots \ldots \ldots$

$\ldots \ldots$

$\ldots \ldots \ldots$

ATTAATTGGA

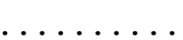

$\ldots \ldots . .$.

...G.....

$\ldots \ldots \ldots$

$\ldots \ldots \ldots$

$\ldots \ldots \ldots$

$\ldots \ldots$

$\ldots \ldots$

$\ldots \ldots \ldots$

TTTTTTCATG

.........

$\ldots \ldots$

$\ldots \ldots \ldots$

$\ldots \ldots \ldots$

$\ldots \ldots \ldots$

$\ldots \ldots \ldots$

$\ldots \ldots$

$\ldots \ldots \ldots$

$\ldots \ldots \ldots$

ATTGGGAGCA

$\ldots \ldots \ldots$

$\ldots \ldots \ldots$

$\ldots \ldots \ldots$

$\ldots \ldots \ldots$

$\ldots \ldots$

$\ldots \ldots \ldots$

$\ldots \ldots \ldots$

$\cdots \cdots \cdots$

CCCTTCTTTA

.........

$\ldots$

$\ldots \ldots$

$\ldots \ldots$

$\ldots \ldots \ldots$

$\ldots \ldots \ldots$

$\ldots \ldots \ldots$

$\ldots \ldots \ldots$

$\ldots \ldots$
GGGACTTCCT ........

$\ldots \ldots \ldots$

$\ldots \ldots \ldots$

$\ldots \ldots$

$\ldots \ldots \ldots$

$\ldots \ldots \ldots$

$\ldots \ldots \ldots$

$\ldots \ldots \ldots$

$\ldots \ldots$

GATGATCAGA

........

.......

.......

$\ldots \ldots$.

........

..A....

$\ldots \ldots$....

.......

$\ldots \ldots \ldots$

GTTATACCAA

.........

$\ldots \ldots \ldots$

$\ldots \ldots \ldots$

$\ldots \ldots \ldots$

$\ldots \ldots \ldots$

$\ldots \ldots \ldots$

$\ldots \ldots \ldots$

$\ldots \ldots \ldots$

$\ldots \ldots \ldots$

cCAgAcAtag

.......

$\ldots \ldots \ldots$

$\ldots \ldots \ldots$

$\ldots \ldots \ldots$

$\ldots \ldots \ldots$

$\ldots \ldots \ldots$

$\ldots \ldots \ldots$

$\ldots \ldots \ldots$

$\ldots \ldots .$.

ACTCTCCTTC

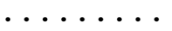

$\ldots \ldots \ldots$

$\ldots \ldots \ldots$

$\ldots \ldots \ldots$

$\ldots \ldots \ldots$

$\ldots \ldots \ldots$

$\ldots \ldots \ldots$

$\ldots \ldots \ldots$

$\ldots \ldots \ldots$ 


\begin{tabular}{|c|c|c|c|c|c|c|}
\hline & A & 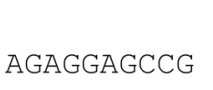 & GTTG & 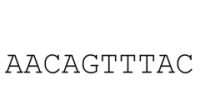 & T & ATCTGGP \\
\hline & $\ldots \ldots$ & 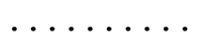 & 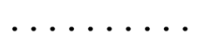 & $\ldots \ldots$ & 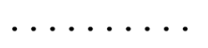 & \\
\hline & $\ldots \ldots$ & $\ldots \ldots$ & 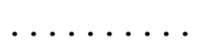 & $\ldots \ldots \ldots$ & 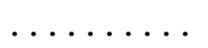 & \\
\hline & $\ldots \ldots \ldots$ & $\ldots \ldots \ldots$ & $\cdots$ & $\ldots \ldots \ldots$ & $\ldots \ldots \ldots$ & 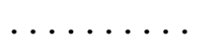 \\
\hline & $\ldots \ldots \ldots$ & $\ldots \ldots \ldots$ & $\cdots$ & $\ldots \ldots \ldots$ & $\ldots \ldots \ldots$ & $\ldots$ \\
\hline & $\ldots \ldots$ & $\ldots \ldots$ & $\cdots$ & $\ldots \ldots \ldots$ & $\ldots \ldots \ldots$ & $\cdots$ \\
\hline & $\ldots \ldots \ldots$ & $\ldots \ldots \ldots$ & . . & $\ldots \ldots \ldots$ & $\ldots \ldots \ldots$ & $\ldots$ \\
\hline & $\ldots G \ldots \ldots$ & $\ldots$ & & $\ldots \ldots \ldots$ & $\ldots \ldots \ldots$ & \\
\hline & $\ldots \ldots \ldots$ & $\ldots \ldots$ & $\ldots$ & $\ldots \ldots \ldots$ & $\ldots \ldots \ldots$ & \\
\hline & 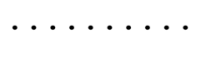 & 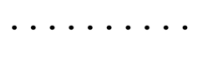 & $\begin{array}{r}\cdots \times \\
\quad 390\end{array}$ & 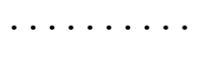 & ( & $\cdots$ \\
\hline & CCCATAGA & GAGCATCTG & AGATTTAGC & AтTTTAGT & TTCATTTAG & TGGTGTA \\
\hline & $\ldots \ldots \ldots$ & $\ldots \ldots$ & $\ldots \ldots \ldots$ & $\ldots \ldots \ldots$ & $\ldots \ldots \ldots$ & $\ldots$ \\
\hline & $\ldots \ldots$ & $\ldots \ldots$ & & $\ldots \ldots \ldots$ & 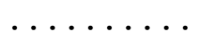 & \\
\hline & $\ldots \ldots \ldots$ & $\ldots \ldots$ & $x^{2}$ & $\ldots \ldots \ldots$ & $\ldots \ldots \ldots$ & $\ldots$ \\
\hline & $\ldots \ldots G$ & $\cdots \cdots \cdots$ & . & $\ldots \ldots \ldots$ & 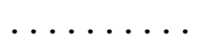 & \\
\hline & $\ldots \ldots \ldots$ & $\ldots \ldots \ldots$ & $\ldots \ldots \ldots$ & $\ldots \ldots \ldots$ & $\ldots \ldots \ldots$ & $\ldots \ldots$ \\
\hline & $\cdots$ & $\cdots$ & & . & . & \\
\hline & $\cdots \cdots \cdots$ & $\cdots \cdots \cdots$ & . & $\cdots$ & es & \\
\hline & $\ldots \ldots \ldots$ & $\ldots \ldots \ldots$ & $\ldots \ldots$ & $\ldots \ldots \ldots$ & $\ldots \ldots \ldots$ & $\ldots$ \\
\hline & & & $\begin{array}{r}\cdots \cdots \\
450\end{array}$ & & & $\cdots \cdots$ \\
\hline & CAAT & AA & ГTTTATTAC & 1 & A & - \\
\hline & $\ldots \ldots$ & $\cdots \cdots$ & $\cdots \cdots \cdots$ & $\ldots \ldots \ldots$ & $\ldots \ldots \ldots$ & $\cdots \cdots$ \\
\hline & $\ldots \ldots \ldots$ & $\ldots \ldots$ & $\ldots \ldots \ldots$ & $\ldots \ldots \ldots$ & $\ldots \ldots \ldots$ & $\ldots \ldots \ldots$ \\
\hline & $\ldots \ldots$ & $\ldots \ldots$ & $\ldots \ldots$ & $\ldots \ldots$ & . & \\
\hline & $\ldots \ldots \ldots$ & $\ldots$ & $\ldots \ldots$ & . &. & \\
\hline & $\ldots$ & $\ldots$ & $\cdots \cdots$ & $\ldots \ldots \ldots$ & $\ldots \ldots \ldots$ & $\ldots$ \\
\hline & $\cdots \cdots \cdots \cdots$ & $\cdots$ & . & $\ldots \ldots \ldots$ & $\ldots \ldots$ & $\ldots \ldots$ \\
\hline & $\ldots \ldots$ & $\cdots$ & $\ldots \ldots$ & $\ldots \ldots$ & $\ldots \ldots$ & $\ldots \ldots$ \\
\hline & $\cdots$ & $\cdots \cdots$ & $\cdots \cdots$ & $\cdots$ & $\cdots$ & \\
\hline & 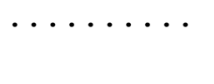 & 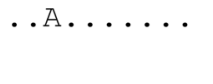 & 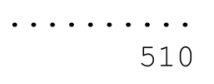 & $\cdots$ & $\cdots$ & $\cdots$ \\
\hline & TAGAA & $\mathrm{C}$ & TGTTTG & GA & TT & TACTT' \\
\hline & $\cdots$ & $\cdots \cdots$ & $\cdots$ & $\cdots \cdots$ & $\ldots \ldots$ & $\ldots \ldots$ \\
\hline & $\cdots$ & $\cdots$ & $\cdot$ & $\cdots$ & $\cdots \cdots$ & $\cdots \cdots$ \\
\hline & $\cdots$ & $\cdots$ & . & & & \\
\hline & - & $\cdots$ & • & $\cdots \cdots$ & $\cdots \cdots \cdots$ & $\cdots$ \\
\hline & • & $\cdots$ & $\cdots$ & $\cdots \cdots$ & $\cdots \cdots$ & 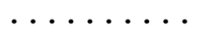 \\
\hline & $\cdots$ & $\cdots$ & & $\cdots$ & $\cdot$ & \\
\hline & . & - & & & $\cdot$ & \\
\hline & $\cdot$ & • & - & $\cdots$ & . & $\cdots$ \\
\hline & $\ldots \ldots \ldots$ & · & $\cdots$ & $\cdots$ & $\ldots$ & $\cdots$ \\
\hline & 1. & $C_{1}$ & CTAT & & & GAA \\
\hline & - & 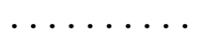 & & $\ldots \ldots$ & $\ldots \ldots \ldots$ & $\cdots$ \\
\hline & . & - & $\cdots$ & $\cdots$ & $\cdot$ & \\
\hline & • & $\cdots$ & & $\cdots$ & $\cdots$ & $\cdots$ \\
\hline & - & $\cdot$ & & & $\cdot$ & \\
\hline & $\cdot$ & $\cdots$ & & & & \\
\hline & & 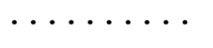 & & $\cdot$ & & \\
\hline & & & & & & \\
\hline & & & & - & & \\
\hline & $\cdots$ & & & & & \\
\hline
\end{tabular}

Fig. 2. continued. 


\begin{tabular}{|c|c|c|c|c|c|c|}
\hline & & & 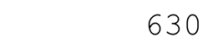 & & & \\
\hline $\mathrm{AR}$ & АСТTCATTT & "TTGATCCGG & CAGGAGGGGG & AGATCCAATT & CTTTATCAAC & ATTTATTT \\
\hline & $\ldots \ldots \ldots$ & $\cdots \cdots$ & . & $\ldots \ldots \ldots$ & $\ldots \ldots \ldots$ & \\
\hline & $\ldots \ldots$ & $\ldots \ldots \ldots$ & $\bullet$ & $\ldots \ldots \ldots$ & $\ldots \ldots \ldots$ & \\
\hline & $\ldots \ldots \ldots$ & $\cdots \cdots \cdots$ & $\cdots$ & $\ldots \ldots \ldots$ & $\ldots \ldots \ldots$ & \\
\hline & $\ldots \ldots$ & $\ldots$ & $\ldots$ & $\ldots \ldots \ldots$ & $\ldots \ldots$ & \\
\hline & $\ldots$ & $\ldots$.... & $\cdots$ & $\ldots$ & $\cdots$ & \\
\hline 07 & $\ldots \ldots \ldots$ & $\ldots \ldots \ldots$ & $\cdots$ & $\ldots \ldots \ldots$ & $\ldots \ldots \ldots$ & \\
\hline & $\ldots \ldots \ldots$ & $\ldots \ldots \ldots$ & 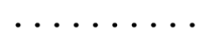 & $\cdots$ & $\cdots$ & \\
\hline & $\ldots \ldots \ldots$ & $\ldots \ldots \ldots$ & $\ldots \ldots F$ & $\ldots \ldots \ldots$ & $\ldots \ldots \ldots$ & $\cdots$ \\
\hline ARSA10 & $\ldots \ldots \ldots$ & $\ldots \ldots \ldots$ & $\ldots G$. . & $\ldots \ldots \ldots$ & $\ldots \ldots \ldots$ & $\cdots$ \\
\hline
\end{tabular}

Fig. 2. continued.

sity within each locality were obtained using Arlequin ver. 3.0 (Excoffier et al., 2005). On the other hand, maximum sequence divergence within population was obtained by extracting the estimate of unrooted pairwise distance within each population from PAUP (Swofford, 2002).

\section{Genetic distance and migration estimate}

Genetic distance and migration rate were estimated from mitochondrial DNA sequences and subroutines in the Arlequin ver. 3.0 (Excoffier et al., 2005). Population pairwise genetic distance $\left(F_{\mathrm{ST}}\right)$ and a permutation test of the significant differentiation of the pairs of localities $(1,000$ bootstraps) were obtained following the approach described in Excoffier et al. (1992) and the distance between DNA sequences were calculated by the Kimura 2-parameters method (Kimura, 1980). Pairwise $F_{\mathrm{ST}}$ values were used to estimate per generation migration rate, $\mathrm{Nm}$ (the product of the effective population size $\mathrm{Ne}$ and migration rate, $m$ ) based upon the equilibrium relationship: $F_{\mathrm{ST}}$ $=1 /(2 N m+1)$.

\section{Genetic structure}

Genetic relationships among populations and sets of populations were assessed by the Holsinger and MasonGamer (H-MG) method (1996). A detailed rationale of this method is described in the original study of Holsinger and Mason-Gamer (1996) and other reports, which utilized this method (Kim et al., 1998). Unlike other variance analyses, this approach generated the hierarchical relationships of the groups without specifying the hierarchical structure of the populations before the analysis (Holsinger and Mason-Gamer, 1996). Therefore, any structure present in the dataset emerged naturally.

Finally, to determine whether any isolation-by-distance (IBD) effect is present in $S$. aterrimus populations, matrices of genetic distance data $\left[F_{\mathrm{ST}} /\left(1-F_{\mathrm{ST}}\right)\right]$ and the logarithms of geographical distance data $(\ln \mathrm{km})$ were constructed among available populations. These matrices were then analyzed to determine their degree of correlation via a Mantel test, with significance tests conducted over 10,000 randomizations (Mantel, 1967). The analysis was conducted using the Isolation-By-Distance software package, with the negative genetic distance set to zero (Bohonak, 2002).

\section{Results and Discussion}

\section{Genetic diversity}

A total of ten haplotypes (BARSA01 - BARSA10) was obtained by sequencing 658-bp of COI gene from 24 individuals of $S$. aterrimus (Fig. 2). Sequence alignment revealed 15 variable nucleotides: 12 transitions (three $\mathrm{T} \rightarrow$ $\mathrm{C}$ and nine $\mathrm{G} \rightarrow \mathrm{A}$ ) and three transversions (two $\mathrm{T} \rightarrow \mathrm{A}$ and

Table 3. Type of substitution in the COI sequences of Scarites aterrimus

\begin{tabular}{ccc}
\hline $\begin{array}{c}\text { Nucleotide } \\
\text { position }\end{array}$ & $\begin{array}{c}\text { Nucleotide } \\
\text { substitution }\end{array}$ & Amino acid substitution \\
\hline 18 & T A & TTT (Phe) TAT (Tyr) \\
\hline 34 & A G & - \\
\hline 94 & A G & - \\
\hline 104 & G A & GAT (Asp) AAT (Asn) \\
\hline 109 & G A & - \\
\hline 186 & T C & TTT (Phe) TCT (Ser) \\
\hline 198 & T C & CTA (Leu) CCA (Pro) \\
\hline 250 & T G & AAT (Asp) AAG (Lys) \\
\hline 269 & C T & CCC (Pro) TCC (Ser) \\
\hline 304 & A G & - \\
\hline 370 & A G & - \\
\hline 433 & G A & - \\
\hline 615 & A T & GAT (Asp) GTT (Val) \\
\hline 625 & A G & - \\
\hline 628 & G A & - \\
\hline
\end{tabular}

-, no amino acid substitution. 
Table 4. Pairwise comparisons among ten haplotypes obtained from COI gene of Scarites aterrimus

\begin{tabular}{cccccccccccc}
\hline & 1 & 2 & 3 & 4 & 5 & 6 & 7 & 8 & 9 & 10 & 11 \\
\hline 1. BARSA01 & - & 0.00152 & 0.00304 & 0.0076 & 0.0076 & 0.00456 & 0.00608 & 0.00608 & 0.00456 & 0.00304 & 0.15957 \\
\hline 2. BARSA02 & 1 & - & 0.00152 & 0.00608 & 0.00608 & 0.00304 & 0.00456 & 0.00456 & 0.00304 & 0.00456 & 0.15805 \\
\hline 3. BARSA03 & 2 & 1 & - & 0.00456 & 0.00456 & 0.00152 & 0.00304 & 0.00304 & 0.00152 & 0.00304 & 0.15653 \\
\hline 4. BARSA04 & 5 & 4 & 3 & - & 0.00912 & 0.00608 & 0.0076 & 0.0076 & 0.00608 & 0.0076 & 0.15957 \\
\hline 5. BARSA05 & 5 & 4 & 3 & 6 & - & 0.00608 & 0.0076 & 0.0076 & 0.00608 & 0.0076 & 0.16109 \\
\hline 6. BARSA06 & 3 & 2 & 1 & 4 & 4 & - & 0.00456 & 0.00456 & 0.00304 & 0.00456 & 0.15805 \\
\hline 7. BARSA07 & 4 & 3 & 2 & 5 & 5 & 3 & - & 0.00608 & 0.00456 & 0.00608 & 0.15957 \\
\hline 8. BARSA08 & 4 & 3 & 2 & 5 & 5 & 3 & 4 & - & 0.00456 & 0.00608 & 0.15653 \\
\hline 9. BARSA09 & 3 & 2 & 1 & 4 & 4 & 2 & 3 & 3 & - & 0.00456 & 0.15502 \\
\hline 10. BARSA10 & 2 & 3 & 2 & 5 & 5 & 3 & 4 & 4 & 3 & - & 0.15957 \\
\hline 11. Loricera pilicornis & 105 & 104 & 103 & 105 & 106 & 104 & 105 & 103 & 102 & 105 & - \\
\hline Num
\end{tabular}

Numbers above the diagonal are mean distance values; numbers below the diagonal are absolute distance values.

one $\mathrm{T} \rightarrow \mathrm{G}$ ) (Table 3). These caused seven amino acid replacements as shown in Table 3.

Pairwise comparison between pairs of haplotypes was performed to know about the divergence and relationships among haplotypes (Table 4). The divergence among ten haplotypes ranged from $0.152 \%$ to $0.912 \%$ (one $\sim$ six nucleotides), indicating moderate to low sequence divergence in this species. In other similar studies, it showed approximately $\leq 1.0 \%$ in a diverse taxonomic group, where homologous region of mitochondrial genome was utilized (Bae et al., 2001; Jeong et al., 2009; Kim et al., 2000; Kim et al., 2007; Kim et al., 2008; Kim et al., 2009; Li et al., 2006). Thus, the magnitude of sequence divergence of the $S$. aterrimus shows moderate to low compared with other insect species.

With regard to distribution BARSA03 showed the widest distribution, accounting for seven of nine localities, composed of $62.5 \%$ ( 15 of 24 ) of samples utilized in this study (Table 1). Except for this haplotype the others are distributed each in one locality represented as a single individual (Table 1). Thus, distribution of $S$. aterrimus haplotypes can be summarized as a restricted local distribution in most haplotypes, except for one haplotype.

\section{Phylogenetic and network analyses}

Phylogenetic analysis was investigated to know evolutionary relationships among haplotypes and to detect any discernable group in connection with geographic distribution (Fig. 3). PAUP analysis by maximum parsimony method showed that all haplotypes were weakly associated or unresolved, with relatively low node support $(\leq$ $44 \%$ ). The network analysis showed that several haplotypes were very closely related to BARSA03 (Fig. 4), evidencing "star phylogeny". Both analyses suggest that $S$. aterrimus haplotypes do not have phylogenetically dis-

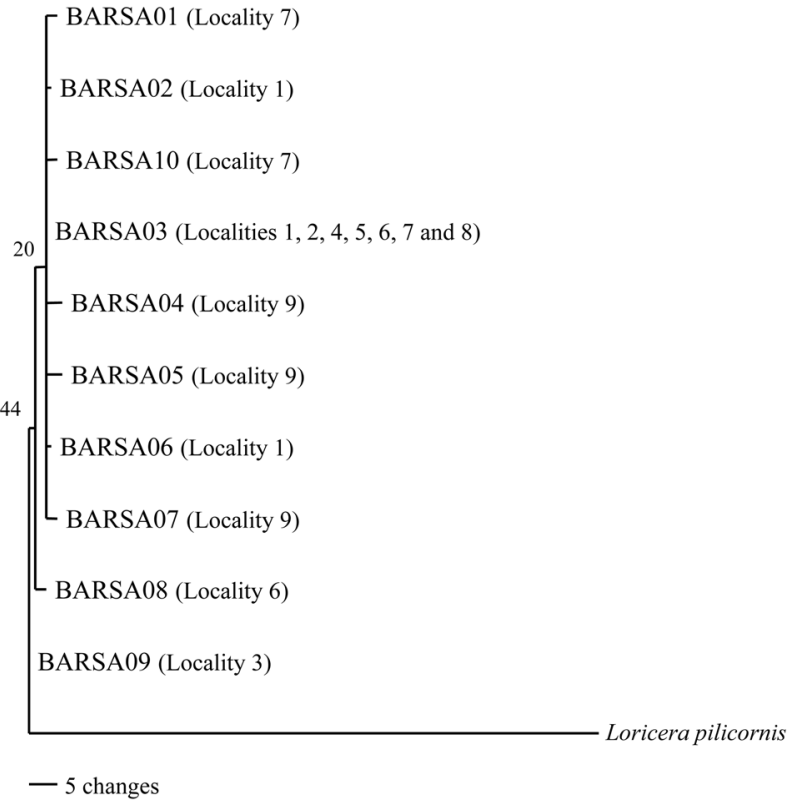

Fig. 3. Phylogenetic analysis of ten haplotypes obtained from mitochondrial COI gene sequence of the Scarites aterrimus. The tree was acquired via the MP method incorporated in PAUP (Phylogenetic Analysis Using Parsimony and Other) ver. 4.0 b10 software (Swofford, 2002). Loricera pilicornis was used as an outgroup in order to root tree. The numbers on the branches represent bootstrap values of 1,000 replications.

tinct or divergent haplotype or haplotype group and this, in turn, suggests that the species occurring in Korean peninsula did not experience historical biogeographic barriers that bolster genetic subdivision.

According to Neigel and Avise (1993), the variance of geographic distribution of a mitochondrial DNA lineage is expected be proportional to its age under a simple isolation by distance model. If this theory is applied to $S$. ater- 


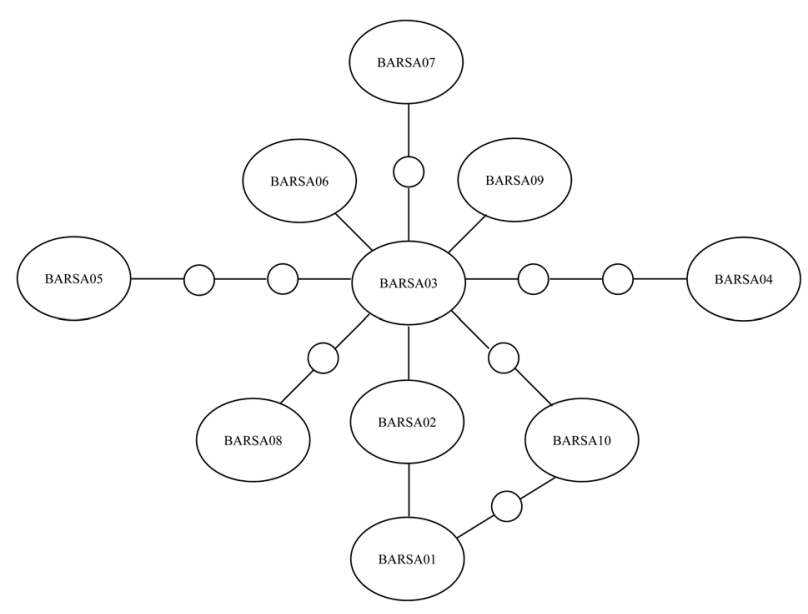

Fig. 4. Parsimonious one-step median networks analysis among ten mitochondrial COI haplotypes of Scarites aterrimus. Each bar represents a single nucleotide difference from the neighboring haplotype. The empty circles indicate missing haplotypes that are not found in this study.

rimus BARSA03 that was found most widely (seven among nine localities with $62.5 \%$ of frequency) seems to be ancestral, and the remaining haplotypes (BARSA01 BARSA02 and BARSA04 BARSA10) found in a single locality might be ones derived from the BARSA03 (Fig. 4). With the consideration of this aspect, it is noteworthy to mention that several haplotypes that are immediately close to BARSA03 were not found in this study, except for three haplotypes, BARSA02, BARSA06, and BARSA09
(Fig. 4). This result indicates that several individuals possessing those missing haplotypes may have not been collected in this study. However, considering that we were unable to collect enough individuals from several localities even with similar trapping effort it is highly likely that those individuals possessing the undiscovered haplotypes may have already been extinct after they have been evolved from the individuals possessing BARSA03. This explanation is plausible in that species that forms a large population size often shows the "star phylogeny", filled with immediately close haplotypes. For example, the mason bee, Osmia cornifrons, has shown a "star phylogeny" fully filled with seven immediately close haplotypes with the centered one that are found most widely and abundantly (Kim et al., 2008). Identically, the bumble bee, Bombus ardens, has also shown a "star phylogeny" fully filled with seven immediately close haplotypes (Kim et al., 2009). In fact, Kim (2003) has investigated species and population diversity of insects dwelling on sand dune habitats in Korean peninsula for 24 years and found only 11 individuals of $S$. aterrimus from four among 16 sand dune areas. This field observation strongly supports a small population of the species and is consistent with the network data

\section{Genetic diversity indices}

The within-locality diversity estimates in terms of haplotype diversity $(H)$, maximum sequence divergence (MSD), mean number of pairwise differences (MPD), and nucleotide diversity $(\pi)$ are presented in Table 5 . In a range of 0

Table 5. Within-locality diversity estimates of Scarites aterrimus

\begin{tabular}{|c|c|c|c|c|c|c|c|}
\hline Locality & $\mathrm{SS}^{\mathrm{a}}$ & $\mathrm{NH}^{\mathrm{b}}$ & $H^{\mathrm{c}}$ & $\mathrm{NP}^{\mathrm{d}}$ & $\operatorname{MSD}^{\mathrm{e}}(\%)$ & $\mathrm{MPD}^{\mathrm{f}}$ & $\pi^{\mathrm{g}}$ \\
\hline 1. Goraebul & 3 & 3 & 1.0000 & 2 & 0.304 & 1.333333 & 0.002026 \\
\hline 2. Dukcheon & 1 & 1 & 1.0000 & 0 & - & - & - \\
\hline 3. Songji & 1 & 1 & 1.0000 & 0 & - & - & - \\
\hline 4. Buldeung & 2 & 1 & 0.0000 & 0 & - & - & - \\
\hline 5. Namryul & 3 & 1 & 0.0000 & 0 & - & - & - \\
\hline 6. Sohwang & 6 & 2 & 0.3333 & 2 & 0.304 & 0.666667 & 0.001013 \\
\hline 7. Songseok & 4 & 3 & 0.8333 & 3 & 0.304 & 1.666667 & 0.002533 \\
\hline 8. Jangpo & 1 & 1 & 1.0000 & 0 & - & - & - \\
\hline 9. Seogwipo & 3 & 3 & 1.0000 & 8 & 0.912 & 5.333333 & 0.008105 \\
\hline
\end{tabular}

${ }^{\text {a Sample size }}$

${ }^{\mathrm{b}}$ Number of haplotypes

cHaplotype diversity

${ }^{\mathrm{d}}$ Number of polymorphic sites

${ }^{\text {e}}$ Maximum sequence divergence

${ }^{\mathrm{f}}$ Mean number of pairwise differences

gNucleotide diversity

-, unavailable. 
$\sim 1$ in $H$, the sand dunes of Seogwipo (locality 9) and that of Goraebul (locality 1) was highest as 1 . In terms of $\pi$, Seogwipo (locality 9) was highest $(\pi=0.008105)$ and the estimates of Goraebul (locality $1 ; \pi=0.002026$ ) and Songseok (locality $7 ; \pi=0.002533$ ) were as low as nearly one quarter of that obtained from Seogwipo (locality 9). Taking these diversity estimates into consideration, the populations in Jeju Island (locality 9) is highest, suggesting that this area supports a larger population than any other regions in Korean peninsula for $S$. aterrimus. For the long-term conservation purpose, this region can be served as a genetic source population to rescue others, which show lower genetic diversity. However, for further decisive conclusion, more scrutinized sampling and molecular analysis involving further rapidly evolving gene may be required.

\section{Gene flow and population structure}

Genetic distance $\left(F_{\mathrm{ST}}\right)$ and per-generation migration rates $(\mathrm{Nm})$ between pairs of populations are shown in Table 6 . Test of statistical significance of pairwise $F_{\text {ST }}$ estimates has shown that only population pair, locality 6 (Sohwang) and locality 9 (Seogwipo), was statistically significant ( $p$ $<0.05$ ), but other pairs of populations, instead, has shown substantial female migration $(\mathrm{Nm}=2.43902 \sim$ infinite $)$. The hierarchical relationships among localities indicated that only locality 6 (Sohwang) has statistically differentiated from the group composed of locality 1 (Goraebul) and locality 9 (Seogwipo), but, in large, these groups are not statistically differentiated from the most basal locality, Songseok (locality 7) (Fig. 5). The results of the Mantel test (10,000 randomizations) for the detection of IBD provided an $r$ (correlation coefficient) value of 0.776 without statistical significance $(p=0.89)$, indicating no positive correlation between geographic distance and genetic distance (Fig. 6). This result can be interpreted as a result of gene flow among $S$. aterrimus populations in Korean pen-

Table 6. Fixation indices $\left(F_{\mathrm{ST}}\right)$ and migration rate $(\mathrm{Nm})$ between pairs of populations of Scarites aterrimus

\begin{tabular}{lrrr}
\hline & 6 & 7 & 9 \\
\hline \multirow{2}{*}{ 1. Goraebul } & $F_{\mathrm{ST}}=0.06897$ & $F_{\mathrm{ST}}=-0.01099$ & $F_{\mathrm{ST}}=0.00000$ \\
\cline { 2 - 3 } & $N m=6.75000$ & $N m=\inf$ & $N m=3752999$ \\
\hline \multirow{2}{*}{ 6. Sohwang } & $F_{\mathrm{ST}}=0.17012$ & $F_{\mathrm{ST}}=0.18182 *$ \\
\cline { 2 - 3 } 7. Songseock & $N m=2.43902 N m=2.25000$ \\
\cline { 2 - 3 } & & $F_{\mathrm{ST}}=0.10048$ \\
\hline
\end{tabular}

$$
\text { 9. Seogwipo }
$$

$* p<0.05$.

inf, infinite.

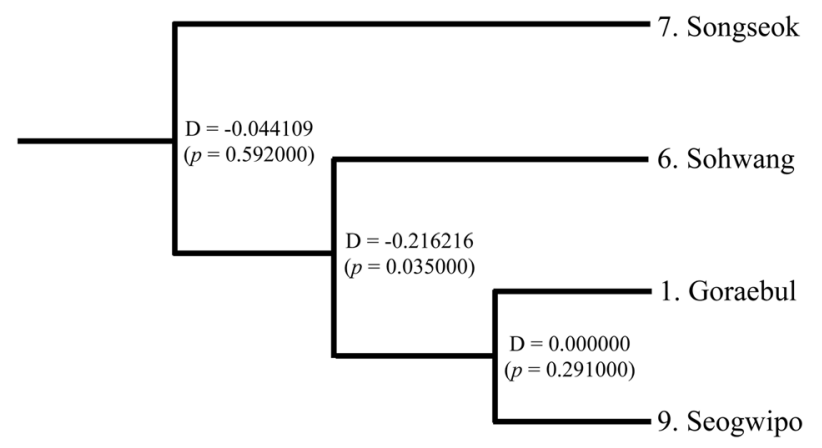

Fig. 5. Hierarchical relationships among localities analyzed using Holsinger and Mason-Gamer's method (1996). The D value is the distance between its two daughter nodes and the $p$ value is the significance of differentiation (based on 10,000 random resamplings).

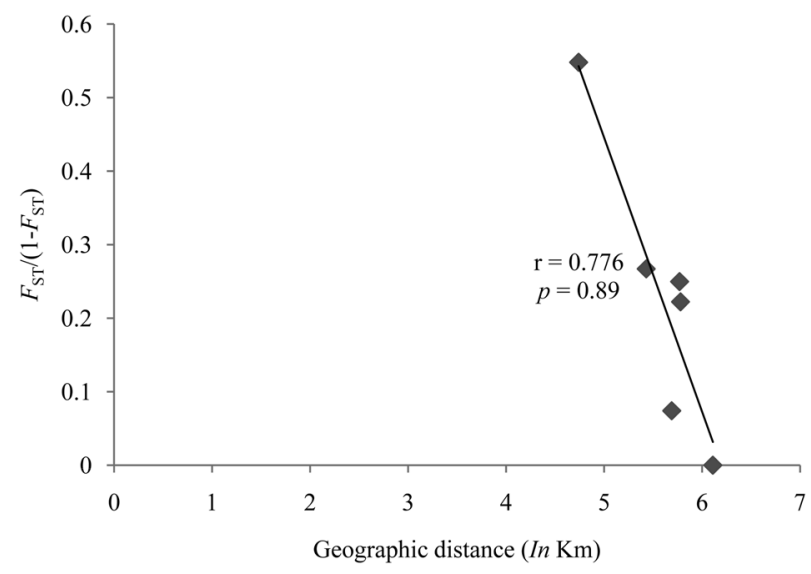

Fig. 6. Scatter plots of genetic distance vs. geographical distance $(l n)$ for pairwise Scarites aterrimus population comparisons based on COI gene sequence.

insula. Collectively, most $S$. aterrimus populations in Korean peninsula do not show genetic structure and are not genetically differentiated, although some divergence of Sohwang population (locality 6) is noteworthy.

Summarized, we collected a total of 24 individuals of $S$. aterrimus from nine Korean localities and sequenced 658 bp of the mitochondrial COI gene. The sequence analysis of $S$. aterrimus provided moderate to low genetic diversity compared with other insect species occurring in Korean peninsula. In terms of geography, Seogwipo population (locality 9) has shown the highest genetic diversity estimates, justifying a prior attention to sustain overall genetic diversity of the species. Considering the result of gene flow and hierarchical population structure, it appears that the geographic populations of $S$. aterrimus evidence a high ratio of gene flow, without long-term zoogeographic barriers. The haplotype network data that provide several undiscovered haplotypes and field observation, such as 
rarity of the species in all sand dunes collectively suggest that the species may have been subjected to severe population decline. For further decisive conclusion, more scrutinized sampling and analysis with further rapidly evolving gene may be required.

\section{Acknowledgement}

This research was supported by the project "Eco Technopia 21" from the Ministry of Environment of Korea (052-091-074 [2009-2011]).

\section{References}

Bae JS, Kim I, Kim SR, Jin BR, Sohn HD (2001) Mitochondrial DNA sequence variation of the mushroom pest flies, Lycoelia mali (Diptera: Sciaridae), and Coboldia fuscipes (Diptera: Scatopsidae), in Korea. App Entomol Zool Jpn 36, 451-457.

Bandelt HJ, Forster P, Sykes BC, Richards MB (1995) Mitochondrial portraits of human populations using median networks. Genetics 141, 743-753.

Bohonak AJ (2002) IBD (Isolation By Distance): a program for analyses of isolation by distance. Heredity 33, 153-154.

Excoffier L, Laval G, Schneider S (2005) Arlequin ver. 3.0. An integrated software package for population genetics data analysis. Evol Bioinf Online 1, 47-50.

Excoffier L, Smouse PE, Quattro JM (1992) Analysis of molecular variance inferred from metric distances among DNA haplotypes: application to human mitochondrial DNA restriction data. Genetics 131, 479-491.

Felsenstein J (1985) Confidence limits on phylogenics: an approach using the bootstrap. Evolution 29, 783-791.

Fitch WM (1971) Toward defining the course of evolution: minimal change for a specific tree topology. Syst Zool 20, 406-416.

Folmer O, Black M, Hoeh W, Lutz R, Vrijenhoek R (1994) DNA primer for amplification of mitochondrial cytochrome c oxidase subunit I from diverse metazoan invertebrates. Mol Mar Biol Biotechnol 3, 294-299.

Hebert PD, Cywinska A, Ball SL, de Waard JR (2003) Biological identification through DNA barcodes. Proc R Soc Lond (B) 270, 313-321.

Holsinger KE, Mason-Gamer RJ (1996) Hierarchical analysis of nucleotide diversity in geographically structured populations. Genetics 142, 629-639.

Jeong HC, Kim JA, Im HH, Jeong HU, Hong MY, Lee JL, Han YS, Kim I (2009) Mitochondrial DNA sequence variation of the swallowtail butterfly, Papilio xuthus, and the cabbage butterfly, Pieris rapae. Biochem Genet 47, 165-178.
Kawakami K, Sugiura S (2006) Feefing of a shore-inhabiting ground beetle, Scarites aterrimus (Coleoptera: Carabidae). Coleopterists Bull 60, 75-79.

Kim JI (1980) Deux nouvelles espèces du genre Psammdius Coréen (Col. Aphodiidae). The Korean J Entomology 5, 9-11.

Kim JI (2003) Insects Fauna from the Coastal Sand-dunes of Korea. Kor J Nature Conser 1, 27-45.

Kim I, Bae JS, Sohn HD, Kang PD, Ryu KS, Sohn BH, Jeong WB, Jin BR (2000) Genetic homogeneity in the domestic silkworm, Bombyx mori, and phylogenetic relationship between $B$. mori and wild silkworm, B. mandarina, using mitochondrial COI gene sequences. Int J Indust Entomol 1, 9-17.

Kim KG, Jang SK, Park DW, Hong MY, Oh KH, Kim KY, Hwang JS, Han YS, Kim I (2007) Mitochondrial DNA sequence variation of the tiny dragonfly, Nannophya pygmaea (Odonata: Libellulidae). Int J Indust Entomol 15, 47-58.

Kim HY, Lee KY, Lee SB, Kim SR, Hong MY, Kim DY, Kim I (2008) Mitochondrial DNA sequence variation of the mason bee, Osmia cornifrons (Hymenoptera: Apidae). Int J Indust Entommol 16, 75-86.

Kim I, Phillips CJ, Monjeau JA, Birney EC, Noack K, Pumo DE, Sikes SR, Dole JA (1998) Habitat islands, genetic diversity, and gene flow in a Patagonian rodent. Mol Ecol 7, 667-678.

Kim MJ, Yoon HJ, Im HH, Jeong HU, Kim MI, Kim SR, Kim I (2009) Mitochondrial DNA sequence variation of the bumblebee, Bombus ardens (Hymenoptera: Apidae). J Asia Pacific Eentomol 12, 133-139.

Kimura M (1980) A simple method for estimating evolutionary rate of base substitution through comparative studies of nucleotide sequences. J Mol Evol 16, 111-120.

Li J, Choi YS, Kim I, Sohn HD, Jin BR (2006) Genetic variation of the diamondback moth, Plutella xylostella (Lepidoptera: Yponomeutidae), in China inferred from mitochondrial COI gene sequences. Euro J Entomol 103, 605-611.

Mantel N (1967) The detection of disease clustering as a generalized regression approach. Cancer Res 27, 209-220.

Neigel JE, Avise JC (1993) Application of a random walk model to geographic distributions of animal mitochondrial DNA variation. Genetics 135, 1209-1220.

Park JK, Paik JC (2001) Economic insects of Korea 12: Coleoptera (Carabidae). Junghaeng-Sa, Suwon, Korea.

Soulé ME (1986) Conservation Biology: the science of scarcity and diversity. Sinauer Associates, Sunderland, MA.

Swofford DL (2002) PAUP* .Phylogenetic Analysis Using Parsimony (*and Other Method) ver. 4.10., Sinauer Associates, Sunderland, MA.

Thomson JD, Gibson TJ, Plewniak F, Jeanmougin F, Higgins DG (1997) The CLUSTAL X windows interface: flexible strategies for multiple sequence alignment aided by quality analysis tools. Nucleic Acids Res 24, 173-216.

Wilson EO (1992) The Diversity of Life. Belknap Press of Harvard Univ, Cambridge, Mass. 